\title{
Indicadores de calidad para unidades de cuidados intensivos respiratorios
}

Quality indicators for respiratory intensive care units

Indicadores de qualidade para as unidades de terapia intensiva respiratórias

Pablo Álvarez-Maldonado, ${ }^{*, \|}$ José Luis Sandoval-Gutiérrez, ${ }^{,, \|}$Víctor Huizar-Hernández, ${ }^{\S, \|}$ Alejandro Hernández-Solís ${ }^{*, \|}$

\section{RESUMEN}

Introducción: Existen indicadores de calidad tanto generales como específicos en medicina crítica. Los indicadores para procesos propios de unidades de cuidados intensivos respiratorios (UCIRs) son necesarios.

Material y métodos: Un grupo de trabajo de las UCIRs de los servicios de tórax de los principales hospitales de tercer y cuarto nivel de atención sanitaria en México, siguiendo la metodología de los grupos de trabajo de la Sociedad Española de Medicina Intensiva, Crítica y Unidades Coronarias y la Fundación Avedis-Donabedian, identificó aspectos relevantes del proceso del cuidado con relación a volumen, riesgo y complejidad, recabando información de 10 años de actividad asistencial para ser correlacionados y finalmente diseñar indicadores. Resultados: Con información de 2,776 pacientes atendidos del $1^{\circ}$ de noviembre de 2009 al $1^{\circ}$ de noviembre de 2019, se delimitó el campo de actuación con aspectos importantes como broncoscopia, neumonía, paciente inmunocomprometido, hemoptisis masiva, embolización de arterias bronquiales, cirugía de resección segmentaria/ lobar o pulmonar, pleurocentesis y ultrasonido fueron identificados y correlacionados en consenso por el grupo de expertos, llevando al diseño de cuatro indicadores.

Conclusiones: Este es el primer reporte de desarrollo de indicadores de calidad para UCIRs de servicios de tórax que servirán para la mejora de la calidad en este tipo de unidades.

Palabras clave: Indicadores, calidad, UCl, tórax, neumología.

\section{ABSTRACT}

Introduction: There are both general and specific quality indicators in critical care medicine. Indicators for respiratory intensive care units (RICUs) processes are needed.

Material and methods: Following the methodology of the working groups of the Spanish Society of Intensive, Critical Care and Coronary Units and the Avedis-Donabedian Foundation, a working group of the RICUs belonging to departments of chest medicine of the main hospitals of third and fourth level of care in Mexico identified relevant aspects of the care process in relation to volume, risk and complexity, collecting information from ten years of clinical activity to be correlated and finally to design indicators.

Results: Delimitation of the field of action was made accounting with information on 2,776 patients treated from November 1, 2009 to November 1, 2019. Important aspects such as bronchoscopy, pneumonia, immunocompromised patient, massive hemoptysis, bronchial artery embolization, segmental/lobar or pulmonary resection surgery, thoracentesis and ultrasound were identified and correlated in consensus by the group of experts leading to the design of four indicators.

Conclusions: This is the first report on the development of quality indicators for RICUs belonging to departments of chest medicine that will serve to improve quality in this type of units.

Keywords: Indicators, quality, ICU, thorax, pulmonology.

\section{RESUMO}

Introdução: Existen indicadores de qualidade tanto gerais como específicos em medicina crítica. São necessários os indicadores para procesos própios das unidades de terapias intensivas respiratórias (UTIRs).

\footnotetext{
* Hospital General de México «Dr. Eduardo Liceaga». Ciudad de México, México.

₹ Instituto Nacional de Enfermedades Respiratorias Ismael Cosío Villegas. Ciudad de México, México.

§ Centro Médico Nacional «La Raza», Instituto Mexicano del Seguro Social. Ciudad de México, México.

"En representación del grupo de trabajo para la revisión, modificación y desarrollo de indicadores de calidad para unidades de cuidados intensivos respiratorios en México y del Capítulo de Cuidados Respiratorios del Colegio Mexicano de Medicina Crítica.

Recibido: 26/06/2020. Aceptado: 21/01/2021.
}

Citar como: Álvarez-Maldonado P, Sandoval-Gutiérrez JL, Huizar-Hernández V, Hernández-Solís A. Indicadores de calidad para unidades de cuidados intensivos respiratorios. Med Crit. 2020;34(6):335-340. https://dx.doi.org/10.35366/98163

www.medigraphic.com/medicinacritica
Material e métodos: Um grupo de trabalho das UTIRs dos serviço de tórax dos principais hospitais de tercero e quarto níveis de atenção sanitária no México, seguindo a metodologia dos grupos de trabalho da Sociedade Espanhola de Terapia Intensiva, Crítica e Unidades Coronárias e a Fundação AvedisDonabedian, identificou aspectos relevantes do processo de cuidado com relação ao volume, risco e complexidade, reunindo informações de 10 anos de atividade assistencial para ser correlacionada e, finalmente, desenhar indicadores.

Resultados: Com informações de 2,776 pacientes atendidos de $1^{\circ}$ de novembro de 2009 a $1^{\circ}$ de novembro de 2019, o campo de atuação foi definido com aspectos importantes como broncoscopia, pneumonia, paciente imunocomprometido, hemoptise maciça, embolização de artérias brônquicas, cirurgia de ressecção segmentar/lobar ou pulmonar, pleurocentese e ultrassom foram identificados e correlacionados em consenso pelo grupo de especialistas, resultando no desenho de quatro indicadores.

Conclusão: Este é o primeiro relatório de desenvolvimento de indicadores de qualidade para UTIRs do serviço de tórax que servirão para melhorar a qualidade neste tipo de unidade.

Palavras-chave: Indicadores, qualidade, UTI, tórax, pneumologia.

\section{INTRODUCCIÓN}

El interés en los procesos de atención médica ocupa un lugar preponderante en la actualidad. ${ }^{1,2}$ La medicina intensiva no escapa del modelo organizacional que puede ser medido en diferentes aspectos y mejorado al identificar áreas de oportunidad. Durante décadas el deseo por mejorar la seguridad de los pacientes ha dado lugar a una serie de iniciativas que buscan implementar sistemas de monitorización estándar para la atención en las unidades de cuidados intensivos (UCls). ${ }^{3-6}$ Uno de los esfuerzos colaborativos de mayor impacto es sin duda el que desarrolla la Sociedad Española de Medicina Intensiva, Crítica y Unidades Coronarias (SEMICyUC) con el soporte metodológico del Instituto Universitario Avedis-Donabedian en España, que propone desde $2005^{7}$ una serie de indicadores de calidad tanto generales como específicos para grupos de pacientes muchas veces atendidos en las UCls especializadas, como es el caso de los portadores de enfermedad de arterias coronarias o de pacientes neurocríticos.

Quienes encabezan los cuidados críticos de los departamentos de medicina del tórax de los principales hospitales de tercer y cuarto nivel de atención sanitaria en México, con el apoyo del Colegio Mexicano de Medicina Crítica (COMMEC) y del Consejo Nacional de Neumología, dieron origen a un proyecto cuyo objetivo fue formular recomendaciones para la monitorización de la calidad en las UCls que por sus características se les ha denominado «unidades de 
cuidados intensivos respiratorios» (UCIRs). ${ }^{8}$ Dentro de sus objetivos se planteó el desarrollo de indicadores de calidad en función de la identificación de procesos clave propios de dichas unidades. El presente trabajo muestra cómo fueron desarrollados indicadores que se proponen para la monitorización de la calidad en las UCIRs.

\section{MATERIAL Y MÉTODOS}

El grupo de trabajo fue conformado durante la 39 Reunión Anual Nacional de la Asociación Mexicana de Medicina Crítica y Terapia Intensiva (hoy COMMEC) en 2012. Sus integrantes, profesionales de las UCIRs de los hospitales de gobierno participantes (la Unidad de Cuidados Intensivos Respiratorios del Servicio de Neumología y Cirugía de Tórax del Hospital General de México, el Departamento de Áreas Críticas del Instituto Nacional de Enfermedades Respiratorias, la Unidad de Cuidados Intensivos Respiratorios del Centro Médico Nacional La Raza y la Unidad de Terapia Intensiva del Servicio de Neumología y Cuidados Intensivos del Hospital Universitario de Monterrey), dieron origen al Capítulo de Cuidados Respiratorios del COMMEC, tomando el proyecto como parte de las actividades del capítulo. Con el paso del tiempo fueron agregados más integrantes de cada sede hospitalaria. Desde el inicio del proyecto hasta la conclusión del desarrollo de los indicadores se dieron un total de ocho reuniones, seis presenciales y dos por vía web a través de videoconferencia.

En la primera reunión se acordaron los objetivos de punto de partida. En la segunda reunión, entre otros, se definió a la UCIR como la UCI dependiente de un Departamento de Medicina del Tórax que atiende a pacientes con patología predominantemente respiratoria en condición crítica, la cual se diferencia de unidades de cuidados intermedios y/o crónicos de retiro de la ventilación mecánica. Las reuniones subsecuentes sirvieron para desarrollar el plan de trabajo y el cronograma, explicar los métodos e identificar y analizar similitudes entre las UCIRs participantes en cuanto a problemas y posibles soluciones, previo a la propuesta de un sistema de monitorización. Los pasos adoptados para el desarrollo de indicadores inmersos en el objetivo de crear un sistema de monitorización comprenden la delimitación del campo de actuación, la identificación de aspectos importantes, la construcción de los indicadores y el establecimiento de los límites de control para cada indicador.

Una vez recabada la información de 10 años de actividad asistencial en relación con demografía, intervenciones, complicaciones, pronóstico y resultados en una UCIR considerada modelo entre sus pares y que además contaba con una base computarizada de recolec- ción de datos prospectivos, ${ }^{9}$ se dio paso en noviembre de 2019 al desarrollo de indicadores de calidad con la identificación y correlación de aspectos importantes y el diseño de los indicadores, siguiendo la metodología empleada por los grupos de trabajo de la SEMICyUC. ${ }^{10}$ Dado que ya existe una diversa gama de indicadores que evalúan la estructura, los procesos y resultados de las UCls en general, la identificación de aspectos importantes se enfocó en procedimientos y protocolos específicos de UCIRs o en gran medida infrecuentes en las UCls polivalentes que implican un gran volumen, riesgo y/o complejidad y por ende son más propios de la medicina toracopulmonar tanto clínica como quirúrgica. En cada aspecto de la atención del enfermo de la UCIR que el grupo de trabajo consideró relevante se vertió un comentario que justifica la selección del proceso antes de dar paso a la etapa de diseño del indicador. El diseño de indicadores que contempla la descripción de diferentes apartados que aseguren su validez y fiabilidad se describe en la Tabla 1.

La evidencia científica que da soporte a cada indicador se obtuvo mediante una búsqueda sistemática en bases de datos electrónicas incluyendo PubMed/ MEDLINE, EMBASE y Cochrane Library hasta el 30 de noviembre de 2019. Después se creó un grupo de cinco expertos que no intervinieron en la etapa de diseño, se contó con la participación de integrantes de cada sede hospitalaria para revisar la definición de cada apartado, lo pertinente de la evidencia científica propuesta y el valor estándar propuesto para cada indicador.

\section{RESULTADOS}

Para la delimitación del campo de actuación se contó con información de 2,776 pacientes atendidos en el periodo del $1^{\circ}$ de noviembre de 2009 al $1^{\circ}$ de noviembre de 2019. El proceso de atención definido como el «cuidado proporcionado al paciente de la UCIR por personal médico y de enfermería especializado» llevó a la

Tabla 1: Descripción breve de los diferentes apartados que aseguran la validez y fiabilidad de los indicadores de calidad.

\begin{tabular}{ll}
\hline Apartado & Definición \\
\hline Dimensión & $\begin{array}{l}\text { Aspecto relevante de la asistencia que se valora en } \\
\text { el indicador } \\
\text { Utilidad del indicador como medida de la calidad. } \\
\text { Se relaciona con la validez; es decir, lo que vamos } \\
\text { a medir ¿tiene sentido? }\end{array}$ \\
& Expresión matemática \\
Fórmula & Definición de términos de la fórmula que puedan \\
Explicación de & ser ambiguos \\
términos & Identificación de la unidad de estudio \\
Población & Estructura, proceso o resultado \\
Tipo & Origen y secuencia para la obtención de datos \\
Fuente de datos & Nivel deseado de cumplimiento del indicador \\
Estándar &
\end{tabular}


identificación de aspectos relevantes que respondieron a tres preguntas clave como se describe a continuación:

a) Volumen: ¿Qué actividades se llevan a cabo con tanta frecuencia que queremos estar seguros de que se llevan a cabo bien?

Se identificaron dos actividades que se llevan a cabo o están presentes con alta frecuencia:

1. Broncoscopia en $10.2 \%$ de los pacientes.

Comentario: uno de cada 10 pacientes es sometido a broncoscopia durante su estancia en la UCIR. La indicación más frecuente para la broncoscopia continúa siendo el diagnóstico de neumonía. Aunque su utilidad diagnóstica es discutida, existen indicaciones como la broncoscopia en el paciente inmunocomprometido en que ésta tiene un papel relevante.

2. Pleurostomía, presente en $29.4 \%$ de los pacientes.

Comentario: una o más sondas pleurales estuvieron presentes en $24.7 \%$ de los pacientes a su ingreso a la UCIR y en otro $4.7 \%$ de los pacientes una sonda pleural fue colocada durante su estancia en la UCIR. La vigilancia y el cuidado de sondas pleurales es de suma importancia para su adecuado funcionamiento. Una disfunción de sonda pleural por oclusión puede llevar a complicaciones que deriven en mayor morbilidad e incluso mortalidad.

b) Riesgo: ¿Qué actividades tienen impacto potencial tan importante en los pacientes que un error sería grave?

Se identificaron dos actividades en que un error en el proceso tendría un impacto grave en la salud de los pacientes ingresados a la UCIR:

1. Pleurocentesis (toracentesis).

Comentario: las complicaciones que derivan de una punción pleural como neumotórax, hemotórax o lesión de órganos intratorácicos o abdominales ponen en riesgo vital a un paciente grave portador de una o más fallas orgánicas, principalmente si la falla es respiratoria o cardiovascular. De ahí que la pleurocentesis deba ser un procedimiento en el que se procure la menor tasa de complicaciones. En la actualidad, existen herramientas que permiten la pleurocentesis con mayor margen de seguridad, una de ellas, de bajo costo, no invasiva y con alto nivel de recomendación, es el ultrasonido.

2. Tratamiento de la hemoptisis masiva.

Comentario: la hemoptisis masiva es una condición derivada de múltiples patologías que puede llevar a la muerte en minutos por ahogamiento, de ahí que el proceso de atención del paciente con hemoptisis debe ser expedito y contar con los tiempos y herramientas necesarias para solucionarlo. Los pacientes con hemoptisis masiva deben ser atendidos siempre que sea posible en centros especializados de hospitales de tercer y cuarto nivel de atención y estos centros a su vez contar con la estructura y personal especializado para dicho propósito.

\section{c) Complejidad: ¿Qué actividades son tan complejas que son propensas a presentar problemas?}

Se identificaron tres actividades: broncoscopia, embolización de arterias bronquiales y cirugía de resección segmentaria/lobar o pulmonar para tratamiento de la hemoptisis masiva.

Comentario: para tener un rendimiento óptimo la broncoscopia es un procedimiento que debe llevarse a cabo por personal capacitado dentro y fuera de la UCI. La broncoscopia es una herramienta diagnóstica y terapéutica valiosa en la hemoptisis masiva que demanda experiencia del operador y material de intervencionismo. Otras dos intervenciones útiles para el tratamiento de la hemoptisis masiva son la «embolización de arterias bronquiales por radiología intervencionista» y la «cirugía de tórax» para la resección del segmento/ lóbulo o pulmón afectados, ambos procedimientos requieren de personal especializado experimentado y son mayormente propios del tercer y cuarto nivel de atención hospitalaria.

Para la construcción de los indicadores se correlacionaron los aspectos importantes de la atención en la UCIR de la siguiente manera:

- Broncoscopia + neumonía + paciente inmunocomprometido

- Hemoptisis masiva + broncoscopia temprana o embolización temprana o cirugía temprana

- Pleurocentesis + complicaciones + ultrasonido

- Pleurostomía + complicaciones + oclusión

En las Tablas 2 a 5 se despliegan los cuatro indicadores con sus respectivos apartados, resultado de la identificación, priorización y correlación de aspectos más relevantes.

\section{DISCUSIÓN}

Los indicadores de calidad son instrumentos de medida que indican la presencia de un fenómeno o suceso y su intensidad, son la unidad básica de un sistema de control y dan paso a las iniciativas de mejora cuando se han identificado problemas o desviaciones de la práctica estandarizada. En el presente trabajo se muestran tres 
indicadores de proceso y uno de resultado propuestos para la monitorización en las UCIRs. En años recientes la aparición de grupos colaborativos ha derivado en la propuesta de indicadores de calidad para grupos específicos de pacientes de las distintas ramas de atención de la medicina crítica que se adapten cada vez más a sus necesidades. ${ }^{11-15}$ Parte del trabajo colaborativo de este grupo consistió en identificar aspectos relevantes de las UCIRs, priorizarlos y construir indicadores reuniendo a un grupo de expertos y con el apoyo logístico de instituciones dedicadas a la medicina respiratoria y del paciente crítico como son el COMMEC y el Consejo
Nacional de Neumología en México. En las sesiones de trabajo se eligieron, por consenso, aquellos indicadores que reunían condiciones de validez (incluyendo su validez aparente), sensibilidad y especificidad. En la actualidad, se dispone de un gran número de indicadores desarrollados por distintas sociedades científicas que son aplicables a las UCls en general, sean especializadas o no. ${ }^{4,7,16} \mathrm{Si}$ bien, el número de indicadores propuesto no es grande, su propósito es cubrir aspectos importantes de la actividad de la especialidad complementando a los sistemas de monitorización ya adoptados por las UCIRs. No obstante, se prevé ampliar el número de

Tabla 2: Indicador número 1.

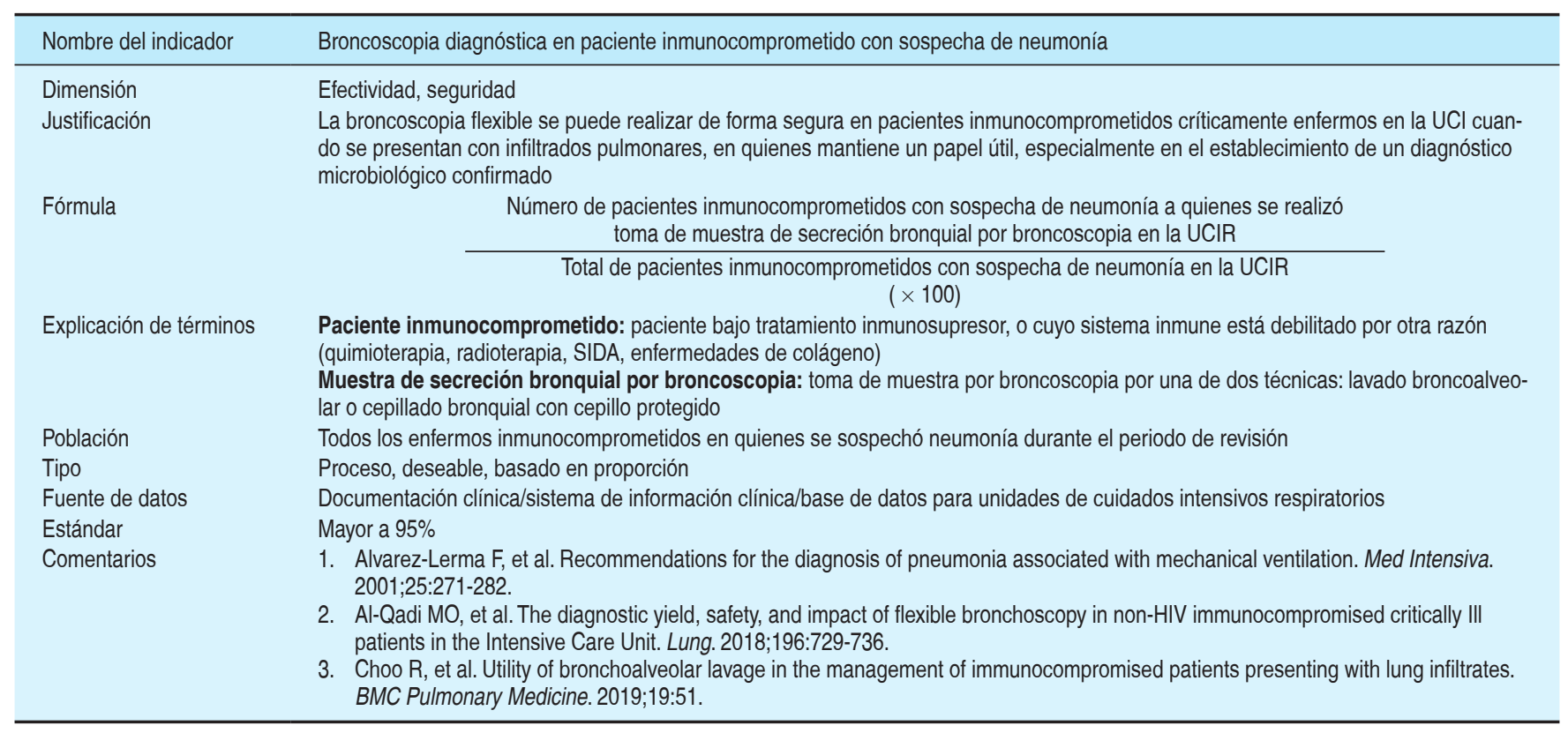

Tabla 3: Indicador número 2.

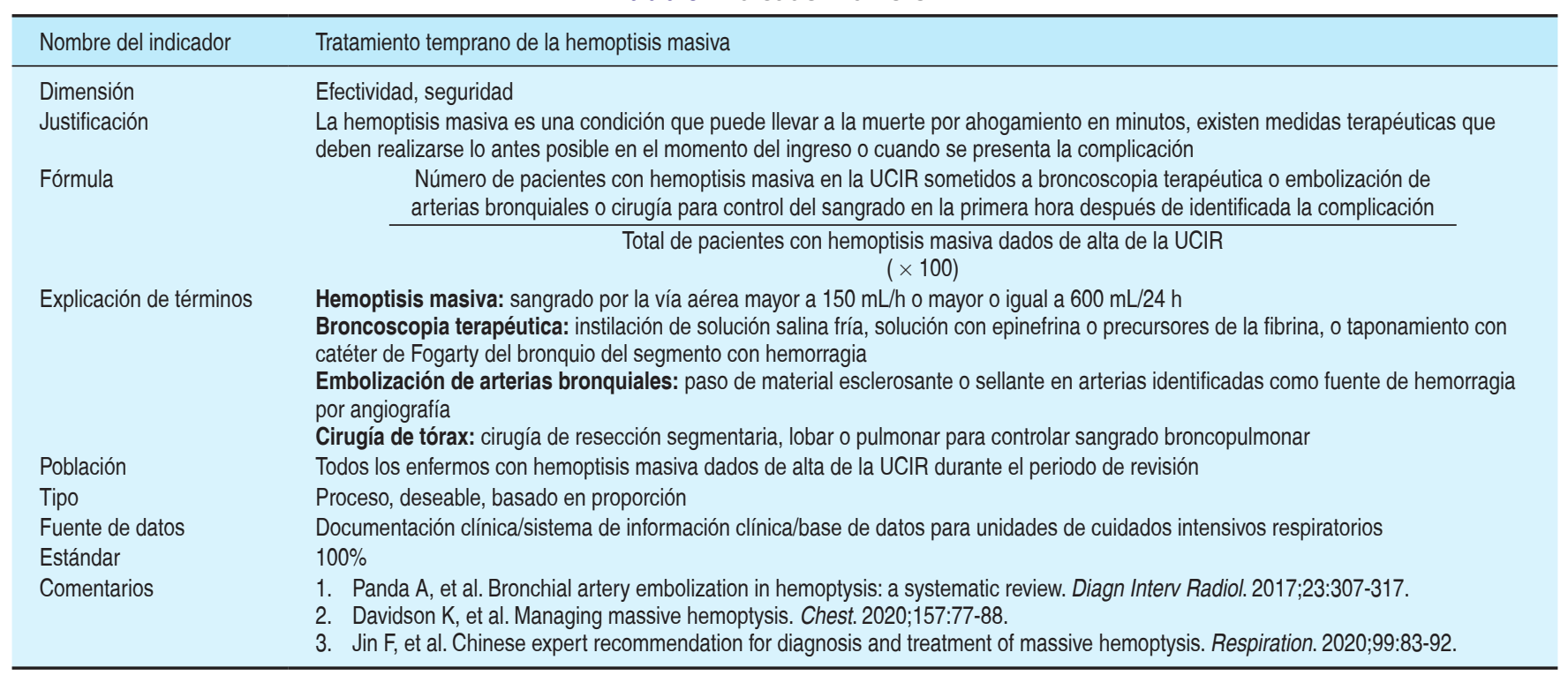


Tabla 4: Indicador número 3.

\begin{tabular}{|c|c|}
\hline Nombre del indicador & Pleurocentesis guiada por ultrasonido \\
\hline Justificación & $\begin{array}{l}\text { Complicaciones relacionadas con la pleurocentesis son más susceptibles de presentarse en pacientes graves en unidades de cuidados } \\
\text { intensivos. El procedimiento requiere de seguridad y efectividad. El guiado de punción con ultrasonido disminuye la tasa de complicacio- } \\
\text { nes por punción pleural }\end{array}$ \\
\hline \multirow[t]{2}{*}{ Fórmula } & Número de pleurocentesis guiada por ultrasonido en la UCIR \\
\hline & $\begin{array}{c}\text { Total de pleurocentesis realizadas en la UCIR } \\
(\times 100)\end{array}$ \\
\hline Explicación de términos & Guía por ultrasonido: guiado en tiempo real de punción con aguja por medio de ultrasonido en dos dimensiones \\
\hline Población & Todos los enfermos a quienes se les realizó pleurocentesis durante el periodo de revisión \\
\hline Tipo & Proceso, deseable, basado en proporción \\
\hline Fuente de datos & Documentación clínica/sistema de información clínica/base de datos para unidades de cuidados intensivos respiratorios \\
\hline Estándar & Mayor a 95\% \\
\hline Comentarios & $\begin{array}{l}\text { 1. Gordon CE, et al. Pneumothorax following thoracentesis: a systematic review and meta-analysis. Arch Intern Med. 2010;170:332- } \\
\text { 339. } \\
\text { 2. Sachdeva A, et al. Thoracentesis and thoracic ultrasound: state of the art in 2013. Clin Chest Med. 2013;34:1-9. } \\
\text { 3. Hooper CE, et al. British Thoracic Society. Pleural procedures and patient safety: a national BTS audit of practice. Thorax. } \\
2015 ; 70: 189-191 \text {. }\end{array}$ \\
\hline
\end{tabular}

Tabla 5: Indicador número 4.

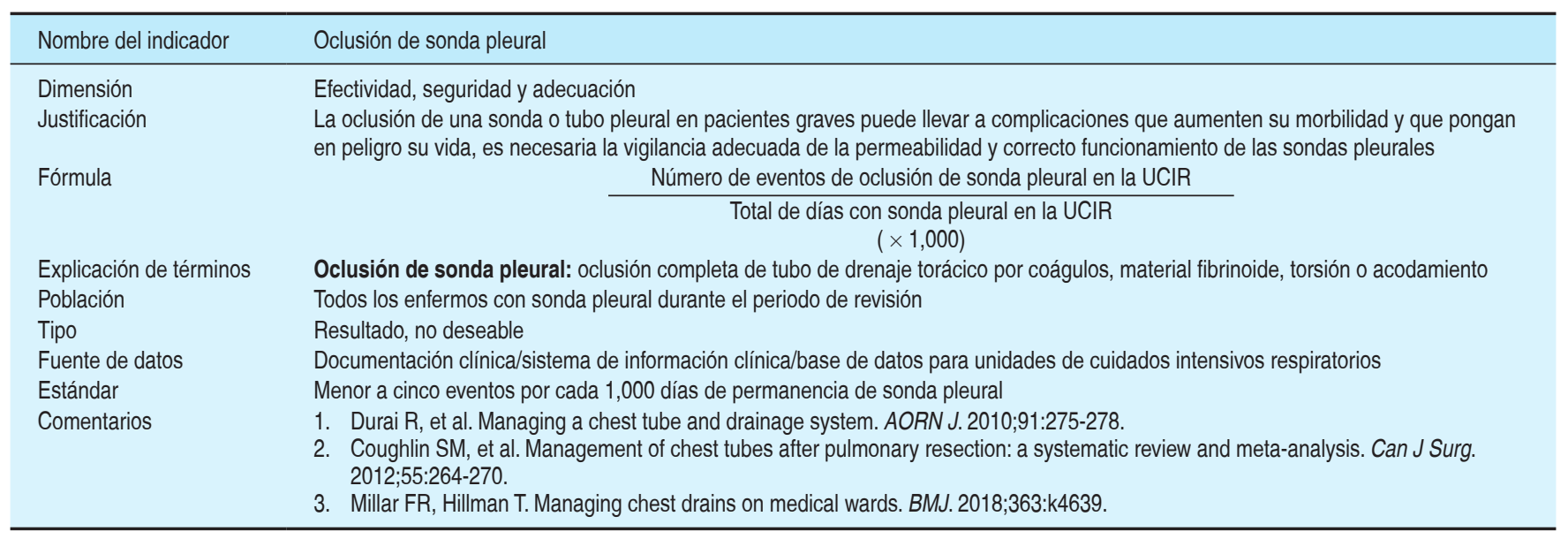

indicadores en el futuro si su implementación resulta práctica y útil.

Otros aspectos no fueron tomados en cuenta durante el diseño de los indicadores. Aunque las UCIRs en México tienen en común que son sitios de referencia en el sistema de salud en situaciones especiales, como lo fue, por ejemplo, la pandemia por el virus de la influenza A H1N1 en 2009 y actualmente lo es la enfermedad por el nuevo coronavirus SARS-CoV-2, éstas constituyen actividades de aparición esporádica o estacional y no de monitorización continua. La disponibilidad en los servicios de tórax de materiales para la broncoscopia simple, la broncoscopia intervencionista, el ultrasonido, una sala de radiología intervencionista y/o quirófanos para la realización de la cirugía torácica de urgencia obviaron la elaboración de indicadores de estructura por considerarse características compartidas entre las UCIRs de los hospitales participantes. Las UCls que deseen adoptar los indicadores propuestos deben considerar estos re- quisitos de estructura, incluyendo al personal de salud especializado. Los indicadores fueron creados pensando en las necesidades de las UCIRs y sus características en común. El cuidado intensivo en el mundo está lejos de ser homogéneo y el concepto de calidad en la UCI puede ser percibido de manera distinta de un país a otro, tal como lo mostró el estudio de Flaatten ${ }^{17}$ en que, de 63 indicadores de calidad evaluados en siete países, ninguno fue común para todos los países. Sin embargo, consideramos que los indicadores propuestos son susceptibles de ser adoptados en cualquier ámbito de atención de la medicina crítica, en el entendido de que el análisis de los resultados deba ser interpretado en el contexto en que han sido evaluados. ${ }^{18}$

Iniciadas las actividades de medida el siguiente paso es evaluar el cumplimiento y grado de control de los indicadores propuestos. El sistema de monitorización no requiere necesariamente de una base de datos computarizada, ya que las variables son fáciles de obtener 
directamente de la documentación clínica o de bitácoras de realización de procedimientos, aunque su futura inclusión en un sistema informático es deseable. La periodicidad de reportes mensuales y el análisis anual parecen ser lo indicado durante las actividades de medida. Tomando en cuenta que es necesaria una validación adicional en pro de recomendar su implementación amplia, se espera que el uso conjunto de los indicadores propuestos en las UCIRs sea dinámico, buscando establecer si la adherencia a los mismos está asociada con mejores resultados para los pacientes.

\section{CONCLUSIONES}

Desde el origen de la medicina crítica como especialidad a mediados del siglo pasado, su avance ha llevado a la diferenciación de UCls para satisfacer las necesidades del tipo de pacientes que atiende con el desarrollo de protocolos y procesos propios de dichas unidades. La medicina toracopulmonar es un ejemplo, con las UCIRs, en que como en otras UCls se busca proporcionar el mejor cuidado, junto con el apoyo de la evidencia disponible, para obtener los mejores resultados. Contar con indicadores de calidad es indispensable para identificar fenómenos que sean susceptibles de cambio con iniciativas de mejora. Este puede considerarse el primer reporte del desarrollo de un grupo de indicadores de calidad para las UCIRs dependientes de departamentos de medicina del tórax, que se espera sean una herramienta útil para programas de mejora de calidad en el futuro en este tipo de unidades.

\section{AGRADECIMIENTOS}

A los doctores Jorge R Sánchez-Medina, José J Elizalde-González, Guillermo Cueto-Robledo, Grisel Hernández-Ríos, Carmen M Hernández-Cárdenas, Víctor M Mendoza-Romero y Raúl Cicero-Sabido, miembros del Colegio Mexicano de Medicina Crítica y del Capítulo de Cuidados Respiratorios por su colaboración y apoyo en el desarrollo del trabajo.

\section{REFERENCIAS}

1. Jha AK. Accreditation, quality, and making hospital care better. JAMA. 2018;320:2410-2411.

2. Shahian DM, Mort EA, Pronovost PJ. The quality measurement crisis: An urgent need for methodological standards and transparency. Jt Comm J Qual Patient Saf. 2016;42:435-438.

3. Chrusch CA, Martin CM, Project TQ. Quality improvement in critical care: selection and development of quality indicators. Can Respir J. 2016;2016:2516765.

4. Curtis JR, Cook DJ, Wall RJ, Angus DC, Bion J, Kacmarek R, et al. Intensive care unit quality improvement: a how-to guide for the interdisciplinary team. Crit Care Med. 2006;34:211-218.

5. Roos-Blom MJ, Dongelmans D, Arbous MS, de Jonge E, de Keizer N. How to assist intensive care units in improving healthcare quality. Development of actionable quality indicators on blood use. Stud Health Technol Inform. 2015;210:429-433.

6. Yang S, Huang LH, Zhao XH, Xing MY, Shao LW, Zhang MY, et al. Using the Delphi method to establish nursing-sensitive quality indicators for ICU nursing in China. Res Nurs Health. 2019;42:48-60.

7. Indicadores de calidad de la SEMICYUC. [Consultado el 8 de febrero de 2020] Disponible en: https://semicyuc.org/indicadoresde-calidad/

8. Álvarez-Maldonado P, Bautista-Bautista E, Huizar-Hernández V, Mercado-Longoria R, Cueto-Robledo G. Grupo de trabajo para la revisión, modificación y desarrollo de indicadores de calidad para unidades de cuidados intensivos respiratorios en México. Arch Bronconeumol. 2015;51:357-358.

9. Álvarez-Maldonado P, Cueto-Robledo G, Cerón-Díaz U, PérezRosales A, Navarro-Reynoso F, Cicero-Sabido R. Indicadores de calidad en una unidad de cuidados intensivos respiratorios. Análisis inicial de la base de datos DEDUCIR. Med Intensiva. 2012;36:518-520.

10. Martín MC, Cabré L, Ruiz J, Blanch L, Blanco J, Castillo F, et al. Indicadores de calidad en el enfermo crítico. Med Intensiva. 2008;32:23-32.

11. Pavesi PC, Guastaroba P, Casella G, Berti E, De Palma R, Di Bartolomeo S, et al. Quality indicators for the assessment of ST-segment elevation acute myocardial infarction (STEMI) networks. How hospital discharge records could be integrated with Emergency medical services data: the Emilia-Romagna STEMI network experience. G Ital Cardiol (Rome). 2015;16:501507.

12. Hoste P, Hoste E, Ferdinande P, Vandewoude K, Vogelaers D, Van Hecke A, et al. Development of key interventions and quality indicators for the management of an adult potential donor after brain death: a RAND modified Delphi approach. BMC Health Serv Res. 2018;18:580.

13. Ignacio E, Mira JJ, Campos FJ, López de Sá E, Lorenzo A, Caballero F. Quality of care and safety indicators in anticoagulated patients with non-valvular auricular fibrillation and deep venous thromboembolic disease. J Healthc Qual Res. 2018;33:68-74.

14. Huijben JA, Wiegers EJA, de Keizer NF, Maas AIR, Menon D, Ercole A, et al. Development of a quality indicator set to measure and improve quality of ICU care for patients with traumatic brain injury. Crit Care. 2019;23:95.

15. Nishimura A, Nishimura K, Onozuka D, Matsuo R, Kada A, Kamitani S, et al. Development of quality indicators of stroke centers and feasibility of their measurement using a nationwide insurance claims database in Japan -J-ASPECT Study-. Circ J. 2019;83:2292-2302.

16. Rhodes A, Moreno RP, Azoulay E, Capuzzo M, Chiche JD, Eddlenton $\mathrm{J}$, et al. Prospectively defined indicators to improve the safety and quality of care for critically ill patients: A report from the Task Force on Safety and Quality of the European Society of Intensive Care Medicine (ESICM). Intensive Care Med. 2012;38:598-605.

17. Flaatten $\mathrm{H}$. The present use of quality indicators in the intensive care unit. Acta Anaesthesiol Scand. 2012;56:1078-1083.

18. Martin Delgado MC. Medir la calidad de la medicina intensiva. Med Intensiva. 2015;39:1-3.

Correspondencia:

Dr. Pablo Álvarez-Maldonado

Servicio de Neumología y Cirugía de Tórax, Torre 503F, Hospital General de México «Dr. Eduardo Liceaga». Dr. Balmis 148, Col. Doctores, Alcaldía Cuauhtémoc, 06720, Ciudad de México, México. E-mail: pamyacs@yahoo.com 